\title{
Contexto alfabetizador familiar: relaciones con la adquisición de habilidades prelectoras y desempeño lector
}

\author{
$\mathrm{M}^{\mathrm{a}}$ Laura Andrés ${ }^{1}$, Sebastián Urquijo ${ }^{1}$, José I. Navarro ${ }^{2}$ y \\ Manuel García-Sedeño ${ }^{2}$ \\ ${ }^{1}$ Universidad Nacional de Mar del Plata (Argentina), ${ }^{2}$ Universidad de Cádiz (España)
}

\begin{abstract}
Algunas habilidades prelectoras pueden preparar al niño para un desempeño lector exitoso. A su vez, la existencia de un contexto alfabetizador familiar favorece la adquisición de estas habilidades prelectoras. El objetivo de este trabajo fue explorar las relaciones entre contexto alfabetizador familiar y adquisición de habilidades prelectoras, así como el estudio de las relaciones entre estas habilidades y el desempeño posterior de la lectura. Para ello se seleccionó una muestra de 52 participantes de 5 años de edad, de clase social media y a los padres o tutores de los niños y niñas. Se aplicó un cuestionario para explorar el contexto alfabetizador familiar a los padres o tutores, una evaluación de habilidades prelectoras mediante la prueba "Prepárate a leer" y una evaluación de procesos lectores a los mismos niños (PROLEC) en el primer curso de educación primaria. Los resultados muestran asociaciones entre el nivel educativo de los padres y la adquisición de habilidades prelectores, así como entre estas y el desempeño lector. La variable con mayor poder explicativo en el desempeño lector fue la conciencia fonológica.
\end{abstract}

Palabras clave: Habilidades prelectoras, contexto alfabetizador familiar, lectura, conciencia fonológica.

Literacy family context. Relationship between pre-reading skills and successful reading. There are pre-reading skills that are able to prepare students for successful reading. At the same time a literacy family context would be improve early reading acquisition. The main goal of this study was to explore the relationship between the literacy family context and early pre-reading skills acquisitions. Other target was study the pre-reading skills and reading performance interaction. 52 kindergarten children, 5 years old, and from a middle social class were used in this study. Their parents were interviewed with a specific questionnaire made for this research in order to know the literacy family context. Participants' pre-reading skills were also assessed by the Spanish version of Get Ready to Read! Screening Tool. And the Spanish version of Reading Processes Assessment Test (PROLEG) was used for reading processes assessment when participants got the first grade. Results show statistically significant relations among parent's education degree and children's pre-reading skills. Significant correlations between reading performance and children's pre-reading skills were also found. The main prediction variable for reading performance was phonological awareness.

Key words: Pre-reading skills, literacy family context, reading, phonological awareness.

Correspondencia: José I. Navarro. Departamento de Psicología, Universidad de Cádiz, Campus Río San Pedro. C.P.: 11510. Puerto Real-Cádiz (España). E-mail: jose.navarro@uca.es 
El aprendizaje de la lectura implica un conjunto de factores que hacen posible su adquisición eficiente. Entre estos factores se destacan el tipo de método pedagógico empleado por el docente (Jiménez, Rodrigo, Ortiz y Guzmán, 1999), las características de los textos elegidos para la enseñanza (Sánchez, 1995), el entrenamiento en los distintos procesos cognitivos lectores (Ramos-Sánchez, 1999), las habilidades y conocimientos prelectores (Piacente, 2005) y el contexto alfabetizador familiar (Querejeta, Piacente, Marder, Resches y Urrutia, 2005).

Por contexto alfabetizador familiar se entiende al conjunto de recursos y experiencias hogareñas en las que participa el niño/a durante los años preescolares, relativos al contacto con eventos de lectura y escritura; y a las interacciones específicas con los adultos de su entorno que favorecen el desarrollo del lenguaje oral y escrito (Graves, Juel y Graves, 2000). Las interacciones específicas son aquellas que tienden a desarrollar habilidades de lenguaje oral y escrito, incluyendo conocimiento conceptual, conocimiento sobre las funciones de la escritura, prácticas de prelectura y habilidades metalingüísticas (Snow y Beals, 2006).

Las habilidades y conocimientos prelectores hacen referencia a un conjunto de precursores de la lectura formal necesarios para tener éxito en el aprendizaje de la lectura que tienen sus orígenes en las experiencias tempranas de la vida de un niño. Existen dos áreas específicas que se encuentran en la base del aprendizaje de la lectura. Los niños/as que desarrollan habilidades potentes en estas áreas tienen mayor éxito en el aprendizaje de la lectura. Se trata del conocimiento de lo impreso y la conciencia lingüística.

El conocimiento de lo impreso abarca el conocimiento que poseen los niños sobre el material gráfico presente en su entorno cotidiano. Incluye el conocimiento del libro, de lo escrito y de algunas letras. El conocimiento del libro se refiere a una comprensión general del libro como objeto. El conocimiento de lo escrito se refiere al conocimiento general que dispone el niño sobre la escritura como sistema, implicando la comprensión de su funcionalidad (Olinghouse y Graham, 2009). Es decir, saber que posee y transmite un significado para un otro distante o ausente; conocer la direccionalidad de la escritura y conocer el aspecto visual de un texto. Por último, el conocimiento de las letras se refiere a conocer el grafismo, el nombre o el fonema que representan algunas letras (Guardia, 2003; Vellutino y Scanlon, 2002).

La conciencia lingüística es definida como la habilidad para reflexionar sobre el lenguaje. Es una habilidad metalingüística porque implica la reflexión y el control deliberado. Incluye la conciencia fonológica y la conciencia léxica. La conciencia fonológica surge en muchas investigaciones como la variable con mayor fuerza predictiva de la lectura inicial (Defior-Citoler, 1994; Stanovich, 2000). Involucra un espectro amplio de habilidades que se desarrollan gradualmente para identificar aspectos sonoros del lenguaje oral, que comienzan operando sobre unidades sonoras mayores tales como la detección de rimas o la segmentación de sílabas- y continúan más 
específicamente con la toma de conciencia sobre la estructura fonémica de las palabras. Mientras el desarrollo de la conciencia fonológica se presenta en interacción con el lenguaje oral, el desarrollo de la conciencia fonémica requiere que el niño interactúe con material escrito. La conciencia léxica consiste en la capacidad para identificar una palabra como un elemento lingüístico separado y como una unidad distinta deslindada del continuo del habla (Borzone, Rosemberg, Diuk, Silvestre y Plana, 2004). Supone la capacidad de aislar las palabras que componen una oración y de reconocer qué palabras cortas están formando otra palabra más larga.

La lectura es una actividad compleja resultante de la interacción de procesos cognitivos de muy diferente nivel, cuya finalidad es decodificar un mensaje escrito y obtener información a partir de él (Maldonado, Sebastián y Soto, 1992). En el aprendizaje de la lectura de una palabra se pueden señalar cuatro procesos principales (Mayer, 2002): (1) el reconocimiento de las unidades grafémicas que la forman; (2) la decodificación de los símbolos e integración de los mismos para formar la palabra; (3) el acceso al significado de esa palabra en la memoria a largo plazo y (4) la integración de la palabra en una frase coherente.

Como se puede apreciar, los factores que inciden en el aprendizaje de la lectura constituyen un grupo diverso y heterogéneo. Distintos estudios han encontrado correlaciones significativas entre el contexto alfabetizador familiar y el desarrollo de habilidades lingüísticas y prelectoras en niños de edad preescolar (Recart-Herrera, Mathiesen-De Gregori y Herrera-Garbarini, 2005).

El nivel socioeconómico del hogar, definido tradicionalmente por el nivel laboral, cultural y de ingresos de la familia (Fernández y Salvador, 1994), posee una asociación significativa con el rendimiento académico de niños en edad escolar (Magnuson, 2007). De hecho, se lo ha estimado como un predictor del desempeño académico de los niños en tareas de lectura y escritura al inicio del primer curso (Nacional Assessment of Educational Progress, 1991). Se considera que la disponibilidad de medios culturales (libros, periódicos, televisión, enciclopedias, etc.) en el hogar es un componente del entorno familiar con una influencia importante en los resultados escolares. Tanto la exposición a material escrito en general como la cantidad de libros presentes en una casa poseen efectos sustanciales en el desarrollo del vocabulario y de habilidades de prelectura en niños de edad preescolar (Echols, West, Stanovich y Zehr, 1996).

El nivel educativo de los padres junto con el tipo de ocupación que poseen, resultan también aspectos importantes para el compromiso en las actividades escolares con sus hijos y, por ende, para su rendimiento (Bracken y Fischel, 2008).

La interacción familiar en lecturas conjuntas tiene un gran impacto sobre el desarrollo del vocabulario, de habilidades de prelectura y sobre el aprendizaje de la lecto-escritura cuando el niño comienza la escolaridad formal. Sobre todo, existe una 
correlación positiva con el tiempo que destinan los padres a estas prácticas y con el grado de compromiso con el que las asumen (Braslavsky, 2004). Ciertas características familiares como el andamiaje y el uso de rutinas facilitan el desarrollo temprano de las habilidades de lectura y escritura. Aquellas familias en las cuales existe un uso cotidiano de la lectura y la escritura -incluyendo comprensión y producción de textos- brindan a sus hijos mayores conocimientos acerca de lo impreso y de la funcionalidad y uso del lenguaje escrito (Purcell-Gates, 1996).

La relación entre las habilidades con las que los niños ingresan al sistema escolar y su rendimiento académico posterior es considerada fuerte y significativa (Baydar, Brooks-Gunn y Furstenberg, 1993). El desempeño en pruebas que evalúan habilidades prelectoras está significativamente relacionado con el desempeño lector (Piacente, 2005). La conciencia fonológica es, dentro de las habilidades prelectoras, uno de los mejores predictores del rendimiento lector y sus componentes, como exactitud, velocidad y comprensión, sobre todo entre los 6 y los 8 años de edad (Vellutino y Scanlon, 2002).

El objetivo de este estudio ha sido explorar las relaciones entre el contexto alfabetizador familiar y la adquisición de habilidades prelectoras y entre estas habilidades prelectoras y el desempeño posterior en pruebas de lectura en una muestra de escolares argentinos de clase social media.

\section{METODO}

\section{Participantes}

La muestra estuvo compuesta por 52 niños y niñas de 5 años de edad que cursaban el tercer curso de Educación Infantil y sus padres o tutores, procedentes de un colegio privado de la ciudad de Mar del Plata (Argentina), de clase social media. De los alumnos, 27 eran niñas $(55.9 \%)$ y 25 eran niños $(48.1 \%)$. De los informantes que completaron el cuestionario, 48 fueron las madres $(92.3 \%)$ y 3 fueron los padres $(7.7 \%)$; el $48.07 \%$ tenía entre 13 y 15 años de escolarización.

\section{Instrumentos}

(1) Contexto alfabetizador familiar. Para la evaluación del contexto alfabetizador familiar se utilizó una entrevista auto administrable, consistente en 20 preguntas de opción múltiple en su mayoría, semiestructurada y precodificada denominada "Evaluación del Contexto Alfabetizador" (Piacente, Querejeta, Marder y Resches, 2003), que explora las siguientes áreas: características del hogar, características de los padres y creencias y prácticas alfabetizadoras. Se facilitaba a los padres y madres de los participantes en el centro escolar y podían cumplimentarla en aproximadamente 30 minutos. 
(2) Habilidades Prelectoras. Para la evaluación de las habilidades prelectoras se utilizó la versión en español del Get Ready to Read! Screening Tool (Whitehurst y Lonigan, 2003). El test se compone de 20 ítems en las que el niño debe señalar una opción entre cuatro posibles y proporciona información sobre los niveles de las habilidades prelectoras. Los distintos niveles se refieren a: habilidades muy básicas (Nivel 1); habilidades que están comenzando a desarrollarse (Nivel 2); habilidades que indican un mayor progreso (Nivel 3); habilidades que indican una preparación medianamente elevada para la lectura (Nivel 4) y habilidades que indican una preparación suficientemente elevada para la lectura (Nivel 5).

(3) Lectura. Para la evaluación de los procesos lectores se utilizó el PROLEC (Cuetos, Rodríguez y Ruano, 1996). La prueba se compone de diferentes sub-escalas que evalúan: conocimiento del nombre o sonido de las letras, reconocimiento de similitudes o diferencias entre pares de palabras, reconocimiento de la diferencia entre palabras y pseudopalabras, lectura de palabras y pseudo palabras balanceadas en cuanto a longitud y estructura semántica y en cuanto a frecuencia de uso en el caso de las palabras, comprensión de oraciones que varían por su estructura gramatical, interpretación de los signos de puntuación en la lectura de un texto breve, comprensión de oraciones y comprensión de textos breves mediante preguntas literales e inferenciales.

\section{Procedimiento}

Se pidió a los padres un consentimiento por escrito para permitir la participación de sus hijos en la investigación. Las pruebas de habilidades prelectoras fueron administradas antes de finalizar el tercer año de la Educación Infantil en forma individual en un aula de la institución destinada para tal fin. A su vez, se les entregó a los padres de los alumnos evaluados la entrevista auto administrable sobre contexto alfabetizador familiar. Los padres realizaban el cuestionario de manera individualizada y posteriormente lo entregaban al profesor de su hijo. Al año siguiente, cuando los niños ya se encontraban cursando el primer grado de la Educación Primaria se les administró la evaluación de procesos lectores PROLEC. La prueba fue administrada por una de las autoras del presente trabajo de manera individualizada, utilizando un formato informático, y asegurándose en cada caso del dominio de las habilidades básicas y capacidad de comprensión de los participantes. La duración de cada sesión de evaluación fue de aproximadamente 60 minutos y se realizó en el entorno escolar, en un aula ofrecida por la institución para tal fin y respetando las condiciones standard de administración de pruebas de evaluación. 


\section{RESULTADOS}

En relación al contexto alfabetizador familiar, la mayoría de los hogares presentaron más de 40 libros de lectura (59.6\%), y el 40.4\% de los hogares presentaron entre 21 y 40 libros específicos para niños. El 69.2\% disponía de televisor y ordenador; y de ellos el $77.7 \%$ permitía al niño utilizar el ordenador. El $42.3 \%$ de los padres nos informaron que leían libros a sus hijos diariamente o casi todos los días. Y un $59.6 \%$ refirieron dibujar y leer con los niños más de dos veces por semana. En relación a los años de escolaridad de los informantes, el $48.07 \%$ tenía entre 13 y 15 años de escolarización. Los años de escolarización de las parejas de los informantes fueron entre 13 y 15 años para un 26.9\%. Del total de los informantes, un $40.4 \%$ no completó los datos relativos a la ocupación que desempeñaban y en un $15.4 \%$ no respondieron por la ocupación de su pareja.

En cuanto a las habilidades prelectoras, la mayoría de los niños (55.8\%) obtuvieron puntuaciones dentro del nivel 5, indicando que se encontraban, al finalizar el tercer año de la Educación Infantil, con una preparación suficientemente elevada para comenzar el aprendizaje de la lectura (tabla 1).

Tabla 1. Niveles de habilidades prelectoras para los niños en el tercer año de Educación Infantil

\begin{tabular}{lccc}
\hline & Frecuencia & $\%$ & $\%$ acumulado \\
\hline $\begin{array}{l}\text { Habilidades que indican un mayor progreso } \\
\text { Habilidades que indican una preparación } \\
\text { medianamente elevada }\end{array}$ & 3 & 5.8 & 5.8 \\
$\begin{array}{l}\text { Habilidades que indican una preparación } \\
\text { suficientemente elevada }\end{array}$ & 20 & 38.5 & 44.2 \\
\hline Total & 29 & 55.8 & 100.0 \\
\hline
\end{tabular}

La tabla 2 presenta los estadísticos descriptivos para el desempeño en las distintas pruebas que evalúan la lectura a partir del test de evaluación de los procesos lectores (PROLEG). Los valores encontrados oscilan entre un valor medio de .019 (DT $=.138$ ) para el dominio de los signos de puntuación (rango 0-1), y $12.384(D T=2.650$, rango 6-15) para el conocimiento de los nombres de las letras.

Los años de escolaridad del informante mostraron relaciones significativas con la disponibilidad de recursos de lectura en el hogar, tanto libros en general como específicos para los niños $(r=.361, p<.01, r=.541, p<.01)$. Los años de escolaridad de la pareja también mostraron correlaciones significativas con la disponibilidad de recursos literarios $(r=.282, p<.05)$. Además, la frecuencia con la que los padres dibujan y escriben con sus niños presentó una correlación significativa con la cantidad de libros disponibles en el hogar $(r=.286, p<.05, r=.317, p<.05)$ y con la frecuencia con la que también leen historias y cuentos con el niño $(r=.411, p<.05)$ (ver tabla 3 ). 
Tabla 2. Medias y desviaciones típicas ( $d t)$ para las pruebas consideradas en el test de evaluación de los procesos lectores (PROLEC)

\begin{tabular}{lcccc}
\hline & Mínimo & Máximo & $M D$ & $D T$ \\
\hline Nombre de letras & 6 & 15 & 12.384 & 2.650 \\
Igual-diferente & 0 & 12 & 9.854 & 2.989 \\
Decisión léxica & 0 & 14 & 4.294 & 5.419 \\
Lectura palabras y pseudo palabras & 0 & 30 & 8.692 & 10.944 \\
Estructuras gramaticales & 0 & 5 & 1.134 & 1.760 \\
Signos de puntuación & 0 & 1 & .019 & .138 \\
Comprensión de oraciones & 0 & 4 & .653 & 1.2662 \\
Comprensión de textos & 0 & 4 & .769 & 1.4904 \\
\hline
\end{tabular}

Tabla 3. Correlaciones no paramétricas ( $r$ de Spearman) para variables de contexto alfabetizador familiar

\begin{tabular}{lcccccc}
\hline & 1 & 2 & 3 & 4 & 5 & 6 \\
\hline 1. Cantidad de libros en el hogar & 1 & $.692^{* *}$ & $.361^{* *}$ & .152 & .165 & $.286^{*}$ \\
2. Cantidad de libros para chicos & & 1 & $.541^{* *}$ & $.282^{*}$ & .188 & $.317^{*}$ \\
3. Años de escolaridad del informante & & & 1 & $.555^{* *}$ & -.054 & .158 \\
4. Años de escolaridad de la pareja & & & & 1 & -.166 & .172 \\
5. Frecuencia con la que se leen cuentos con el niño & & & & & & $.411^{* *}$ \\
6. Frecuencia con la que se dibuja y escribe con el niño & & & & & & 1 \\
\hline$* * 0.01$ (bilateral). ${ }^{*} p<.05$ (bilateral). & & & &
\end{tabular}

Se realizó la prueba de Kruskal-Wallis para observar diferencias en el rendimiento en habilidades prelectoras teniendo en cuenta la cantidad de años de escolaridad de los cuidadores. Se apreciaron diferencias significativas en las habilidades prelectoras de acuerdo a la cantidad de años de escolaridad $\left(X_{(2 \mathrm{gl})}^{2}=11.003, p<.01\right)$ (tabla 4).

Tabla 4. Prueba de Kruskal-Wallis para contrastar medias de rendimiento en habilidades prelectoras y años de escolaridad de los padres informantes

\begin{tabular}{lcccccc}
\hline & $\begin{array}{c}\text { Años de escolaridad } \\
\text { de los padres }\end{array}$ & $N$ & Rango promedio & \multirow{2}{*}{$X^{2}$} & gl & Sig. asintótica \\
\cline { 2 - 4 } \multirow{3}{*}{$\begin{array}{l}\text { Habilidades } \\
\text { prelectoras }\end{array}$} & $14-9$ & 13 & 17.79 & & \\
\cline { 2 - 4 } & $10-12$ & 13 & 22.50 & 2 & $.004 * *$ \\
\cline { 2 - 4 } & $13-15$ & 25 & 33.46 & & & \\
\hline
\end{tabular}

Variable de agrupación: Años de escolaridad del informante. $* * p<.01$

Para analizar qué grupos difieren entre sí se aplicó la prueba U de MannWhitney para dos muestras independientes. Se utilizó la corrección de Bonferroni para controlar la tasa de errores tipo I $(p<.017)$. La diferencia entre el grupo que poseía entre 7 y 9 años de escolaridad y aquel que poseía entre 13 y 15 años resultó significativa $(U=72, p<.017)$. 
Tabla 5. Estadísticos para la prueba $U$ de Mann-Whitney de contrastes entre medias según años de escolaridad del informante

\begin{tabular}{|c|c|c|c|c|c|c|c|c|}
\hline & $\begin{array}{c}\text { Años de } \\
\text { escolaridad de los } \\
\text { padres }\end{array}$ & $N$ & $\begin{array}{l}\text { Rango } \\
\text { promedio }\end{array}$ & $\begin{array}{l}\text { Suma } \\
\text { de } \\
\text { rangos }\end{array}$ & $\begin{array}{c}\text { Ude } \\
\text { Mann- } \\
\text { Whitney }\end{array}$ & $\begin{array}{c}\text { Wde } \\
\text { Wilcoxon }\end{array}$ & Z & $\begin{array}{c}\text { Sig. } \\
\text { asintótica } \\
\text { (bilateral) }\end{array}$ \\
\hline \multirow{3}{*}{$\begin{array}{l}\text { Habilidades } \\
\text { prelectoras }\end{array}$} & Entre 7 y 9 años & 14 & 12.64 & 177 & \multirow{3}{*}{72} & \multirow{3}{*}{177} & \multirow{3}{*}{-3.046} & \multirow{3}{*}{$.002 *$} \\
\hline & Entre 13 y 15 años & 25 & 24.12 & 603 & & & & \\
\hline & Total & 39 & & & & & & \\
\hline
\end{tabular}

a Variable de agrupación: Años de escolaridad del informante. * $p<.017$

Tabla 6. Matriz de correlaciones entre conocimiento de lo impreso, conciencia lingüística y procesos lectores

\begin{tabular}{|c|c|c|c|c|c|c|c|c|c|c|}
\hline & 1 & 2 & 3 & 4 & 5 & 6 & 7 & 8 & 9 & 10 \\
\hline $\begin{array}{l}\text { 1. Conocimiento de lo } \\
\text { impreso }\end{array}$ & 1 & $.358 * *$ & $.335^{*}$ & .260 & .194 & $.295^{*}$ & $.348^{*}$ & .106 & .234 & .269 \\
\hline 2. Conciencia Lingüística & & 1 & $.455^{* *}$ & -.10 & $.544 * *$ & $.587 * *$ & $.318^{*}$ & .175 & $.507 * *$ & $.431 * *$ \\
\hline 3. Nombre de letras & & & 1 & .105 & $.543^{* *}$ & $.555^{* *}$ & $.510 * *$ & .140 & $.438^{* *}$ & $.445 * *$ \\
\hline 4. Tarea igual-diferente & & & & 1 & .096 & .135 & .184 & .007 & .005 & .032 \\
\hline 5. Decisión Léxica & & & & & 1 & $.923 * *$ & $.804 * *$ & .229 & $.781 * *$ & $.748 * *$ \\
\hline $\begin{array}{l}\text { 6. Lectura palabras y } \\
\text { pseudo palabras }\end{array}$ & & & & & & 1 & $.791 * *$ & .159 & $.799 * *$ & $.758 * *$ \\
\hline 7. Estructuras gramaticales & & & & & & & 1 & .150 & $.699 * *$ & $.647 * *$ \\
\hline 8. Signos de puntuación & & & & & & & & 1 & .039 & .212 \\
\hline $\begin{array}{l}\text { 9. Comprensión de } \\
\text { oraciones }\end{array}$ & & & & & & & & & 1 & $.830 * *$ \\
\hline 10. Comprensión de textos & & & & & & & & & & 1 \\
\hline
\end{tabular}

Tabla 7. Coeficientes de las habilidades prelectoras para el análisis de regresión lineal en el desempeño en lectura

\begin{tabular}{|c|c|c|c|c|c|c|}
\hline \multirow{2}{*}{\multicolumn{2}{|c|}{ Modelo }} & \multicolumn{2}{|c|}{$\begin{array}{l}\text { Coeficientes no } \\
\text { estandarizados }\end{array}$} & \multirow{2}{*}{$\begin{array}{c}\begin{array}{c}\text { Coeficientes } \\
\text { estandarizados }\end{array} \\
\beta\end{array}$} & \multirow[t]{2}{*}{$t$} & \multirow[t]{2}{*}{ Sig. } \\
\hline & & $\beta$ & Error típ. & & & \\
\hline \multirow[t]{5}{*}{1} & (Constante) & -20.072 & 19.391 & & -1.035 & .306 \\
\hline & Conocimiento del libro & 6.206 & 5.903 & .119 & 1.051 & .299 \\
\hline & Conocimiento de algunas letras & 2.207 & 4.401 & .068 & .501 & 618 \\
\hline & Conocimiento de lo escrito & 4.719 & 7.150 & .086 & .660 & .513 \\
\hline & Conciencia fonológica & 8.505 & 1.591 & .621 & 5.345 & .000 \\
\hline
\end{tabular}

a Variable dependiente: Desempeño total en lectura

Se obtuvo una correlación estadísticamente significativa $(r=.568, p<.001)$ entre el rendimiento total en las habilidades prelectoras $(X=15.63, D T=2.65)$ y el desempeño total en lectura $(X=36.96, D T=22.70)$, las habilidades prelectoras de conocimiento de lo impreso y conciencia lingüística también obtuvieron correlaciones significativas con los distintos procesos lectores evaluados con el PROLEC (tabla 6).

Finalmente, para conocer el peso estadístico de las diferentes habilidades prelectoras evaluadas en el desempeño total en lectura se realizó un análisis de regresión 
lineal. El modelo resultó significativo. La variable que resultó con mayor capacidad predictiva del desempeño en lectura fue la conciencia fonológica $(\beta=8.505, p<.001)$ (tabla 7).

\section{DISCUSION}

El objetivo de este trabajo fue estudiar las relaciones entre el contexto alfabetizador familiar y la adquisición de habilidades prelectoras en un grupo de escolares argentinos al finalizar el tercer año de la Educación Infantil, y entre estas habilidades prelectoras y el desempeño en lectura cuando ya se encontraban en el primer curso de la Educación Primaria.

Los resultados indican que aquellos padres que poseen mayor cantidad de años de escolaridad presentan hogares en los que se reportan mayor disponibilidad de recursos vinculados a la lectura, como libros en general y libros de lectura para niños en particular; mayores prácticas relativas a la lectura y escritura como leer, dibujar y escribir con sus hijos, coincidiendo con resultados de otros estudios (Recart-Herrera et al., 2005; Braslavsky, 2004).

Hemos encontrado en este estudio, en aquellos hogares en los que los padres poseen mayor cantidad de años de escolarización, se observaron los mayores niveles de desempeño en habilidades prelectoras por parte de sus hijos. Estos hogares suelen ofrecer a sus hijos mayor cantidad de recursos y experiencias relativos al contacto con situaciones de lectura y escritura, e interacciones específicas como lecturas conjuntas que favorecen el desarrollo del lenguaje oral y escrito (Graves et al., 2000). Por otro lado, el desarrollo de habilidades prelectoras se relacionó positivamente con el desempeño en lectura. Específicamente la conciencia fonológica fue la variable con mayor capacidad predictiva en los desempeños en lectura, coincidiendo con otros autores (Piacente, 2005; Vellutino y Scanlon, 2002). Las palabras familiares tienen acceso directo al léxico por lo que se utiliza preferentemente la ruta léxica, mientras que las palabras desconocidas tienen un acceso indirecto a través de un proceso de recodificación fonológico (Rueda, 1995). Para poder leer a través de la ruta fonológica, el lector tiene que ser capaz de segmentar el habla en sus fonemas componentes, o sea, debe tener desarrollada la conciencia fonológica. En la lectura en español, los niños utilizan las dos rutas. Los principiantes utilizan predominantemente la vía fonológica y progresivamente van pasando a la ruta visual. Esto podría explicar por qué aquellos niños con mayores desarrollos de la conciencia fonológica logran resultados superiores en las pruebas que evalúan lectura al inicio de los primeros aprendizajes. Las diferencias en el nivel de desarrollo de la conciencia fonológica de los prelectores predice diferencias posteriores en la facilidad y rapidez para aprender las habilidades básicas de decodificación de palabras (Domínguez, 1996). 
De este estudio y de investigaciones previas, se desprende que enseñar a los niños a percibir la conciencia fonológica en educación infantil podría ayudarles a aprender a leer posteriormente, y será de gran ayuda para los niños que tienen problemas en este aprendizaje (Bryant y Bradley, 1998).

Además, se han encontrando resultados favorables para la adquisición de habilidades prelectoras y para el aprendizaje de la lectura en aquellas actividades compartidas con familiares que involucran lectura frecuente de libros de cuentos (Borzone, 1996); manipular materiales impresos (Martín, 1996); o buscar regularidades entre fonología y ortografía (Sánchez, 1996). Por lo que la implementación de programas de intervención destinados a incrementar la adquisición de estas habilidades de prelectura en niños de Educación infantil, a través de actividades de lectura conjuntas con sus padres, resulta recomendable y ha obtenido consecuencias favorables en el desempeño lector posterior de los niños (Pearson, Bair, Kamil y Mosenthal, 1991).

Debe recordarse, sin embargo, que numerosos factores intervienen en el aprendizaje lector eficaz: la competencia auditiva, la atención visomotora, la habilidad matemática, la inteligencia general, (Leppanen, Niemi, Aunola y Nurmi, 2004), el método pedagógico empleado, las características de los textos elegidos y el entrenamiento en los distintos procesos cognitivos lectores (Jiménez et al., 1999) entre otros. Por lo que la intervención temprana en Educación Infantil no parece ser suficiente por sí sola para prevenir fracasos en el desempeño lector posterior. Resulta recomendable que las intervenciones continúen inclusive en los cursos posteriores (Saint-Laurent y Giasson, 2001).

\section{Agradecimientos}

Parcialmente realizado con la financiación del proyecto de investigación: SEJ200762420/EDUC.

\section{REFERENCIAS}

Baydar, N., Brooks-Gunn, J. y Furstenberg, F.F. (1993). Early warning signs of functional illiteracy: Predictors in childhood and adolescence. Child Development, 64, 815-829.

Borzone, A.M. (1996). Aprender a leer y a escribir a los 5. Aportes a la Educación Inicial. Buenos Aires: Aique.

Borzone, A.M., Rosemberg, C.R., Diuk, B., Silvestre, A. y Plana, D. (2004) Niños y maestros por el camino de la alfabetización. Buenos Aires: Red de Apoyo Escolar.

Bracken, S.S. y Fischel, J.E. (2008). Family reading behavior and early literacy skills in preschool children from low-income backgrounds. Early Education and Development, 19, 45-67.

Braslavsky, B. (2004). ¿Primeras letras o primeras lecturas? Una introducción a la alfabetización temprana. Buenos Aires: Fondo de Cultura Económica.

Bryant, P. y Bradley, L. (1998). Psicología de la lectura. Madrid: Alianza Editorial.

Cuetos, F. Rodríguez, B. y Ruano, E. (1996). Prueba de evaluación de los procesos lectores (PROLEC). Madrid: TEA. 
Defior-Citoler, S.A. (1994). La conciencia fonológica y la adquisición de la lectoescritura. Infancia y Aprendizaje, 67-68, 91-113.

Domínguez, A. B. (1996). El desarrollo de habilidades de análisis fonológico a través de programas de enseñanza. Infancia y Aprendizaje, 76, 69-81.

Echols, L.D., West, R.F., Stanovich, K.E. y Zehr, K.S. (1996). Using children's literacy activities to predict growth in verbal cognitive skills: A longitudinal investigation. Journal of Educational Psychology, 88, 296-304.

Fernández, S. y Salvador, F. (1994). La familia ante el fracaso escolar. Educadores, 36, 7-22.

Graves, M., Juel, C. y Graves, B. (2000). Teaching reading in the 21 st century (3rd ed.). Needham Heights, MA: Allyn \& Bacon.

Guardia, P. (2003). Relaciones entre habilidades de alfabetización emergente y la lectura desde el nivel de transición mayor a primero básico. Psykhé, 12, 63-79.

Jiménez, J.E., Rodrigo, M., Ortiz, M.R. y Guzmán, R. (1999). Dossier Documental. Procedimientos de evaluación e intervención en el aprendizaje de la lectura y sus dificultades desde una perspectiva cognitiva. Infancia y Aprendizaje, 88, 107-122.

Leppanen, U., Niemi, P., Aunola, K. y Nurmi, J.E. (2004). Development of reading skills among preschool and primary school pupils. Reading Research Quarterly, 39, 72-93.

Magnuson, K. (2007). Maternal education and children's academic achievement during middle childhood. Developmental Psychology, 43, 1497-1512.

Maldonado, A., Sebastián, E. y Soto, P. (1992). Retraso en lectura: evaluación y tratamiento educativo. Madrid: Ediciones de la Universidad Autónoma de Madrid.

Martín, B. (1996). Automatización del acceso léxico: ¿es posible desde el lenguaje integrado? Cultura y Educación, 2, 103-113.

Mayer, R.E. (2002). Psicología de la educación. El aprendizaje en las áreas de conocimiento. Madrid: Pearson.

National Assessment of Educational Progress, (1991). The 1989-90 national assessment of reading and literature. Denver, CO: Author.

Olinghouse, N.G. y Graham, S. (2009). The relationship between the discourse knowledge and the writing performance of elementary-grade students. Journal of Educational Psychology, 101, 37-50.

Pearson, P.D., Bair, M., Kamil, M. y Mosenthal, P. (1991). Handbook of reading research. Nueva York: Longman.

Piacente, T. (2005). Las habilidades y conocimientos prelectores, su incidencia en el aprendizaje formal. En J. Vivas (Ed.) Las Ciencias del Comportamiento en los albores del Siglo $X X I . X R A A C C$. Mar del Plata: UNMDP.

Piacente, T., Querejeta, M. Marder, S. y Resches, M. (2003). Evaluación del Contexto Alfabetizador. La Plata: Comisión de Investigaciones Científicas (Inédito. Mimeo).

Purcell-Gates, V. (1996). Stories, coupons, and the TV Guide: Relationship between home literacy experiences and emergent literacy knowledge. Reading Research Quarterly, 31, 406428.

Querejeta, M., Piacente, T., Marder, S., Resches, M. y Urrutia, M.I. (2005). Características del contexto alfabetizador en familias de diferente nivel socioeconómico. Un estudio comparativo. En E. Diez-Villoria, B. Zubiauz y M.A. Mayor (Eds.), Estudio sobre la Adquisición del Lenguaje (pp. 803-818) Salamanca: Universidad de Salamanca.

Ramos-Sánchez, J.L. (1999). Una perspectiva cognitiva de las dificultades lectoescritoras. Procesos de evaluación e intervención. Madrid: Ciudad Nueva.

Recart-Herrera, M.I., Mathiesen-De Gregori, M.E. y Herrera-Garbarini, M.O. (2005). Familia del preescolar y su desempeño escolar posterior. Revista Enfoques Educacionales, 7, 105123. 
Rueda, M.I. (1995). La lectura. Adquisición, dificultades e intervención. Salamanca: Amarú

Saint-Laurent, L. y Giasson, J. (2001). Effects of a multicomponents literacy program and of supplement phonological session on at-risk kindergartners. Educational Research and Evaluation, 7, 1-33.

Sánchez, M. (1995). Los textos expositivos. Estrategias para mejorar su comprensión. Buenos Aires: Santillana.

Sánchez, M. (1996). El todo y las partes: una crítica al lenguaje integrado. Cultura y Educación, 1, 39-54.

Snow, C.E. y Beals, D.E. (2006). Mealtime talk that supports literacy development. New Directions for Child and Adolescents Development, 111, 51-66.

Stanovich, K.E. (2000). Progress in understanding reading. Scientific foundationes and new frontiers. New York: The Guilford Press.

Vellutino, F.R. y Scanlon, D.M. (2002). Emergency literacy skills, early instruction and individual differences as determinants or difficulties in learning to read: The case for early intervention. En S. Neuman y D. Dickinson (Eds.), Handbook of early literacy research (pp. 295-321). Londres: The Guildford Press.

Whitehurst, G.J. y Lonigan, C. (2003). Get Ready to read! An early literacy manual: screening tool, activities and resources. USA: Pearson Early Learning.

Recibido: 4 de julio de 2009 Recepción Modificaciones: 20 de diciembre de 2009

Aceptado: 10 de enero de 2010 